\title{
Non-Hermitian extension of gauge theories and implications for neutrino physics
}

\author{
Jean Alexandre, ${ }^{a}$ Carl M. Bender ${ }^{a, b}$ and Peter Millington ${ }^{c}$ \\ ${ }^{a}$ Department of Physics, King's College London, \\ Strand WC2R 2LS, London, United Kingdom \\ ${ }^{b}$ Department of Physics, Washington University, \\ One Brookings Drive, St. Louis, MO 63130, U.S.A. \\ ${ }^{c}$ Physik Department T70, Technische Universität München, \\ James-Franck-Straße, 85748 Garching, Germany \\ E-mail: jean.alexandre@kcl.ac.uk, cmb@wustl.edu, p.w.millington@tum.de
}

ABSTRACT: An extension of QED is considered in which the Dirac fermion has both Hermitian and anti-Hermitian mass terms, as well as both vector and axial-vector couplings to the gauge field. Gauge invariance is restored when the Hermitian and anti-Hermitian masses are of equal magnitude, and the theory reduces to that of a single massless Weyl fermion. An analogous non-Hermitian Yukawa theory is considered, and it is shown that this model can explain the smallness of the light-neutrino masses and provide an additional source of leptonic $\mathcal{C P}$ violation.

Keywords: Neutrino Physics, Gauge Symmetry, Space-Time Symmetries

ARXIV EPRINT: 1509.01203 


\section{Contents}

1 Introduction 1

2 Description of the Model 3

2.1 General description 3

2.2 Chiral basis 4

2.3 Hidden gauge invariance $\quad 6$

2.4 Exceptional points 8

3 One-loop corrections $\quad 9$

4 Implications for neutrino masses 11

5 Conclusions $\quad 16$

$\begin{array}{ll}\text { A One-loop corrections } & 17\end{array}$

\section{Introduction}

Discrete symmetries play a fundamental role in particle physics. Charge conjugation $(\mathcal{C})$ and the discrete spacetime symmetries of parity $(\mathcal{P})$ and time reversal $(\mathcal{T})$ are such that $\mathcal{C P} \mathcal{T}$ is necessarily conserved for a local, Lorentz-symmetric, and Hermitian theory. There is, however, no reason for all of the latter requirements to be essential in the building of viable models. This has been shown, for example, in ref. [1], where locality is dropped, leading to a Lorentz-symmetric description of neutrino physics, which is odd under $\mathcal{C P} \mathcal{T}$. In the present article we keep locality but consider a non-Hermitian (Lorentzsymmetric) model.

The last 15 years have seen much interest in and research activity on theories described by non-Hermitian Hamiltonians. Such theories have remarkable and often unexpected properties. For example, the eigenvalues of the non-Hermitian $\mathcal{C P} \mathcal{T}$-symmetric quantum-mechanical Hamiltonians $H=p^{2}+i x^{3}$ and $H=p^{2}-x^{4}$ are real, positive, and discrete $[2,3]$.

One idea that has been pursued repeatedly is to study the properties of a non-Hermitian version of quantum electrodynamics (QED). The Hamiltonian for QED becomes nonHermitian if the unrenormalized electric charge $e$ is chosen to be imaginary. Then, if the electric potential is chosen to transform as a pseudovector rather than a vector, the Hamiltonian becomes $\mathcal{C P} \mathcal{T}$ symmetric. The resulting non-Hermitian theory of electrodynamics becomes a multi-component analog of a self-interacting spinless quantum field theory (QFT), comprising a pseudoscalar field $\phi$ with a cubic self-interaction term of the form $i \phi^{3}$. This pseudoscalar QFT was studied in detail in ref. [4], and the non-Hermitian version of QED was studied in ref. [5]. This non-Hermitian version of electrodynamics is 
particularly interesting because it is asymptotically free and the version of this theory with massless fermions appears to have a nontrivial fixed point (see refs. [6-8]). A perturbative calculation of a metric with respect to which this theory is unitary is given in ref. [9].

A detailed analysis of $\mathcal{C P} \mathcal{T}$-symmetric non-Hermitian fermionic theories was done by Jones-Smith and Mathur [10]. In this work it was emphasized that for fermions the timereversal operator $\mathcal{T}$ has the property that $\mathcal{T}^{2}=-1$. This represents a significant departure from the case of bosonic theories, where $\mathcal{T}^{2}=1$. (Further work on the properties of $\mathcal{C P} \mathcal{T}$ symmetric representations of fermionic algebras may be found in ref. [11].) In addition, Jones-Smith and Mathur showed that free noninteracting $\mathcal{C P} \mathcal{T}$-symmetric Dirac equations have the remarkable feature that massless neutrinos can exhibit species oscillations [12].

The discovery of neutrino oscillations and the observation of the baryon asymmetry of the universe (BAU) (see ref. [13]) have been driving forces in the study of the neutrino sector of the SM. Neutrino oscillations consistent with experimental observations can occur if the SM neutrinos have small but finite masses. The misalignment of the mass and flavour eigenbases then gives rise to the PMNS [14, 15] mixing matrix, analogous to the CKM $[16,17]$ mixing matrix of the quark sector. In order to generate the BAU, it is necessary to satisfy the Sakharov conditions [18]: namely the presence of out-of-equilibrium dynamics and the violation of baryon number $B$, charge $\mathcal{C}$, and charge-parity $\mathcal{C P}$. Both the CKM and PMNS matrices contain a complex phase, which provides a source of $\mathcal{C P}$ violation in the SM. In the quark sector this gives rise to the $\mathcal{C P}$ violation observed in $K-, D-, B$ - and $B_{s}$-meson mixing (see ref. [13]). However, the magnitude of this $\mathcal{C P}$ violation is insufficient to have generated the observed BAU. An elegant framework in which both experimental observations may be accommodated is provided by the scenario of leptogenesis [19] (for reviews, see refs. [20-23]). Therein, the SM is supplemented with heavy Majorana neutrinos. The smallness of the light neutrino masses arises by means of the see-saw mechanism [24-28] and the baryon asymmetry through the decays of the heavy neutrinos in the expanding early universe. By virtue of the lepton-number $L$-violating Majorana mass terms and complex Yukawa couplings, which provide an additional source of $\mathcal{C}$ and $\mathcal{C P}$ violation, these decays are able to generate an initial lepton excess, which is subsequently converted to a baryon excess via the $(B+L)$-violating electroweak-sphaleron interactions of the SM [29].

In this article we examine an extension of QED that involves the usual Dirac mass term $m \bar{\psi} \psi$ and an anti-Hermitian mass term $\mu \bar{\psi} \gamma^{5} \psi$. The fermion field is coupled to the photon through both vector and axial-vector couplings. The anti-Hermitian mass term is separately $\mathcal{C}$ even, $\mathcal{P}$ odd and $\mathcal{T}$ even, and is consistent with unitarity for $\mu^{2} \leq m^{2}$. We study the gauge symmetry of this model and show that, although gauge invariance is lost in the massive case, it is recovered in the specific situation where the Hermitian and antiHermitian mass terms have equal amplitude $\mu^{2}=m^{2}$. In this limit we find that the model reduces to that of a massless left- or right-chiral Weyl fermion. Moreover, we illustrate that by choosing the ratio $\mu / m$ we may obtain an arbitrarily small but finite mass for the fermion and give more or less prominence to one chirality. This observation, combined with the maximal $\mathcal{C P}$ violation of the anti-Hermitian mass term, may be directly relevant to neutrino physics. 
The paper is organized as follows: section 2 begins by summarizing the essential properties of the free non-Hermitian fermion theory studied already in refs. [30] and [31]. Subsequently, the gauge interactions are introduced and the tree-level properties of the model are described. Therein, emphasis is given to the restoration of gauge invariance in the limit $\mu^{2}=m^{2}$. Section 3 presents the one-loop self-energy and vertex corrections, the details of which are given in appendix A. Here, the recovery of gauge invariance is made explicit through the expected vanishing of the longitudinal component of the vacuum polarization. Section 4 describes an analogous non-Hermitian Yukawa model and discusses possible implications for the neutrino sector of the SM. A novel mechanism for generating the light neutrino masses as well as the presence of an additional source of $\mathcal{C P}$ violation is highlighted. Concluding remarks are given in section 5 .

\section{Description of the Model}

\subsection{General description}

We begin with the free fermion non-Hermitian Lagrangian considered in ref. [30]:

$$
\mathcal{L}_{0}=\bar{\psi}\left(i \not \partial-m-\mu \gamma^{5}\right) \psi,
$$

with $\mu^{2} \leq m^{2}$, such that the energies $\omega$ are real for all three-momenta $\vec{p}$; that is,

$$
\omega^{2}=\vec{p}^{2}+M^{2} \geq 0,
$$

where

$$
M^{2}=m^{2}-\mu^{2} .
$$

It is shown in ref. [31] that the conserved current for this model is

$$
j^{\rho}=\bar{\psi} \gamma^{\rho}\left(1+\frac{\mu}{m} \gamma^{5}\right) \psi
$$

and that the equation of motion is obtained by taking the variation of the action with respect to $\bar{\psi}$ for fixed $\psi$. The anti-Hermitian mass term in eq. (2.1) is even under both charge conjugation $\mathcal{C}$ and time-reversal $\mathcal{T}$, and odd under parity $\mathcal{P}$. Thus, it is odd under $\mathcal{C P} \mathcal{T}$. However, this does not contradict invariance under Lorentz-symmetry, since Hermiticity has been relaxed.

In this article we gauge this model and include both vector and axial-vector coupling to an Abelian $\mathrm{U}(1)$ gauge field $A_{\mu}$ :

$$
\mathcal{L}=-\frac{1}{4} F^{\mu \nu} F_{\mu \nu}+\bar{\psi}\left[i \not \partial-\not A\left(g_{V}+g_{A} \gamma^{5}\right)-m-\mu \gamma^{5}\right] \psi,
$$

where $F_{\mu \nu}=\partial_{\mu} A_{\nu}-\partial_{\nu} A_{\mu}$. In the massless case $m=\mu=0$ the action is invariant under the combined vector and axial gauge transformation

$$
\begin{aligned}
A_{\mu} & \longrightarrow A_{\mu}-\partial_{\mu} \phi, \\
\psi & \longrightarrow \exp \left[i\left(g_{V}+g_{A} \gamma^{5}\right) \phi\right] \psi, \\
\bar{\psi} & \longrightarrow \bar{\psi} \exp \left[i\left(-g_{V}+g_{A} \gamma^{5}\right) \phi\right] .
\end{aligned}
$$

However, in the massive case $m \neq 0$ and/or $\mu \neq 0$ this gauge invariance is lost. 
The free fermion propagator of this theory is

$$
i S=i \frac{\not p+m-\mu \gamma^{5}}{p^{2}-M^{2}+i \varepsilon}
$$

where $\varepsilon=0^{+}$. We see immediately that eq. (2.7) has a light-like pole for $\mu= \pm m\left(M^{2}=0\right)$, like that of a massless theory, with the propagator taking the form

$$
i S=i \frac{\not p+m\left(\mathbb{I}_{4} \mp \gamma^{5}\right)}{p^{2}+i \varepsilon} .
$$

The mass term in the numerator is proportional to the chiral projection operators

$$
P_{R(L)}=\frac{1}{2}\left(\mathbb{I}_{4}+(-) \gamma^{5}\right)
$$

where $\mathbb{I}_{n}$ is the $n \times n$ unit matrix. Separating the right- and left-chiral components $\psi_{R}=$ $P_{R} \psi$ and $\psi_{L}=P_{L} \psi$ in the current (2.4), we see that the probability density may be written as

$$
\rho=\left(1+\frac{\mu}{m}\right)\left|\psi_{\mathrm{R}}\right|^{2}+\left(1-\frac{\mu}{m}\right)\left|\psi_{\mathrm{L}}\right|^{2} .
$$

Evidently, for $\mu=+(-) m$ the contribution to the probability density is entirely from the right-(left)-handed degree of freedom. Therefore, it appears that in the limit $\mu=+m$ we obtain a massless right-handed theory, and in the limit $\mu=-m$ we obtain a massless left-handed theory. This feature is the focus of this article. Moreover, in section 4, we comment on potential implications of this non-Hermitian theory for the neutrino sector of the SM and, in particular, the smallness of the light-neutrino masses.

The preceding observations suggest that it proves illustrative to consider this theory in an explicit chiral basis. We do so in the following section and show explicitly that invariance under the gauge transformation in eq. (2.6) is recovered in the limit $\mu \rightarrow \pm m$, as we would anticipate for a theory that appears to be effectively massless.

\subsection{Chiral basis}

In order to recast eq. (2.5) in an explicit chiral basis, we first rotate from the Dirac basis to the Weyl basis via the orthogonal transformation

$$
\psi_{W}=\left(\begin{array}{c}
\psi_{L} \\
\psi_{R}
\end{array}\right)=\frac{1}{\sqrt{2}}\left(\begin{array}{cc}
\mathbb{I}_{2} & -\mathbb{I}_{2} \\
\mathbb{I}_{2} & \mathbb{I}_{2}
\end{array}\right) \psi .
$$

We may then work directly with the two-component right- and left-chiral spinors $\psi_{R}$ and $\psi_{L}$.

In the Weyl basis the gamma matrices take the form

$$
\gamma_{W}^{\mu}=\left(\begin{array}{cc}
0 & \sigma^{\mu} \\
\bar{\sigma}^{\mu} & 0
\end{array}\right), \quad \gamma_{W}^{5}=\left(\begin{array}{cc}
-\mathbb{I}_{2} & 0 \\
0 & \mathbb{I}_{2}
\end{array}\right)
$$


where $\sigma^{\mu}=\left(\sigma^{0}, \sigma^{i}\right)$ and $\bar{\sigma}^{\mu}=\left(\sigma^{0},-\sigma^{i}\right)$, and $\sigma^{i}$ are the Pauli matrices. To avoid a proliferation of subscripts and superscripts, throughout this paper we suppress $\mathrm{SU}(2)$ spinor indices (see appendix A). In addition, the projection operators are given by

$$
P_{L}=\left(\begin{array}{cc}
\mathbb{I}_{2} & 0 \\
0 & 0
\end{array}\right), \quad P_{R}=\left(\begin{array}{cc}
0 & 0 \\
0 & \mathbb{I}_{2}
\end{array}\right) .
$$

The fermionic sector Lagrangian is then

$$
\mathcal{L}_{\text {ferm }}=\left(\begin{array}{ll}
\psi_{L}^{\dagger} & \psi_{R}^{\dagger}
\end{array}\right)\left(\begin{array}{cc}
i \bar{\sigma} \cdot D_{-} & -m_{+} \\
-m_{-} & i \sigma \cdot D_{+}
\end{array}\right)\left(\begin{array}{l}
\psi_{L} \\
\psi_{R}
\end{array}\right),
$$

where

$$
m_{ \pm}=m \pm \mu
$$

and the covariant derivatives are given by

$$
D_{ \pm}^{\mu}=\partial^{\mu}+i g_{ \pm} A^{\mu}
$$

with

$$
g_{ \pm}=g_{V} \pm g_{A}
$$

Notice that $\gamma^{5}$ matrices no longer appear explicitly in the Lagrangian eq. (2.14). Instead, the non-Hermitian nature of this theory is manifest in the asymmetry between the rightand left-chiral components of the original four-component Dirac spinor.

We may study the on-shell structure of the Lagrangian in eq. (2.14). For the case $\mu=+m$ the Lagrangian takes the form

$$
\left.\mathcal{L}_{\text {ferm }}\right|_{\mu=+m}=\psi_{L}^{\dagger} i \bar{\sigma} \cdot D_{-} \psi_{L}+\psi_{R}^{\dagger} i \sigma \cdot D_{+} \psi_{R}-2 m \psi_{L}^{\dagger} \psi_{R},
$$

giving the following equations of motion for $\psi_{R}$ and $\psi_{L}$ :

$$
\begin{aligned}
& \frac{\delta S}{\delta \psi_{R}^{\dagger}}=0 \quad \Rightarrow \quad i \sigma \cdot D_{+} \psi_{R}=0 \\
& \frac{\delta S}{\delta \psi_{L}^{\dagger}}=0 \quad \Rightarrow \quad i \bar{\sigma} \cdot D_{-} \psi_{L}=2 m \psi_{R} .
\end{aligned}
$$

Since the left-chiral field does not appear in the equation of motion for the right-chiral field [eq. (2.19a)], we may integrate it out, giving the tree-level on-shell Lagrangian

$$
\mathcal{L}_{\text {on-shell }}^{\text {tree }}=\psi_{R}^{\dagger} i \sigma \cdot D_{+} \psi_{R},
$$

which describes a massless theory of right-handed Weyl fermions. This is precisely what we saw in the probability density [eq. (2.10)]. Moreover, the on-shell Lagrangian [eq. (2.20)] respects the full vector and axial-vector gauge invariance [see eq. (2.6)]; that is,

$$
A_{\mu} \longrightarrow A_{\mu}-\partial_{\mu} \phi, \quad \psi_{R} \longrightarrow \exp \left(i g_{+} \phi\right) \psi_{R}
$$

For the case $\mu=-m$ we need only make the replacements $\psi_{R} \leftrightarrow \psi_{L}, \bar{\sigma} \leftrightarrow \sigma$, and $D_{+} \leftrightarrow D_{-}$in eq. (2.20), yielding a massless theory of left-handed Weyl fermions. The next subsection gives a more explicit argument to justify the restoration of gauge invariance for the light-like case $\mu^{2}=m^{2}$. 


\subsection{Hidden gauge invariance}

In this subsection we show that gauge invariance is recovered when $\mu^{2}=m^{2}$. To do so, we construct a two-component spinor basis in which gauge invariance is explicit.

Written in block form, where the LL (left-left) element is located in the top left $2 \times 2$ block, the mass matrix is given by

$$
\boldsymbol{M}=\left(\begin{array}{cc}
0 & m_{+} \\
m_{-} & 0
\end{array}\right),
$$

having eigenvalues $\pm M= \pm \sqrt{m^{2}-\mu^{2}}$ and eigendirections

$$
e_{ \pm}=\frac{1}{\sqrt{2}}\left(\begin{array}{c} 
\pm x_{+} \\
x_{-}
\end{array}\right) \quad \text { with } \quad x_{ \pm} \equiv \sqrt{1 \pm \mu / m}
$$

We rotate to the mass eigenbasis but first allow for a rescaling of the left- and right-handed components:

$$
\psi_{L(R)} \longrightarrow \psi_{L(R)}^{\prime}=a_{L(R)} \psi_{L(R)},
$$

where $a_{L(R)}$ are to be determined later, as explained below. This leads to the transformation

$$
\left(\begin{array}{l}
\psi_{+} \\
\psi_{-}
\end{array}\right) \equiv \boldsymbol{V}^{-1}\left(\begin{array}{l}
\psi_{L} \\
\psi_{R}
\end{array}\right)
$$

with

$$
\boldsymbol{V}^{-1}=\frac{1}{\sqrt{2}}\left(\begin{array}{cc}
a_{L} x_{-} & a_{R} x_{+} \\
-a_{L} x_{-} & a_{R} x_{+}
\end{array}\right) \quad \text { and } \quad \boldsymbol{V}=\frac{1}{\sqrt{2}}\left(\begin{array}{cc}
1 /\left(a_{L} x_{-}\right) & -1 /\left(a_{L} x_{-}\right) \\
1 /\left(a_{R} x_{+}\right) & 1 /\left(a_{R} x_{+}\right)
\end{array}\right)
$$

The Lagrangian then becomes

$$
\begin{aligned}
\mathcal{L}_{\mathrm{ferm}} & =\left(\psi_{+}^{\dagger} \psi_{-}^{\dagger}\right) \boldsymbol{V}^{\boldsymbol{\top}}\left(\begin{array}{cc}
i \bar{\sigma} \cdot D_{-} & -m_{+} \\
-m_{-} & i \sigma \cdot D_{+}
\end{array}\right) \boldsymbol{V}\left(\begin{array}{c}
\psi_{+} \\
\psi_{-}
\end{array}\right) \\
& =\left(\psi_{+}^{\dagger} \psi_{-}^{\dagger}\right)\left(\begin{array}{cc}
A & B \\
C & D
\end{array}\right)\left(\begin{array}{c}
\psi_{+} \\
\psi_{-}
\end{array}\right),
\end{aligned}
$$

where

$$
\begin{aligned}
A & =\frac{i \sigma \cdot D_{+}}{2 a_{R}^{2} x_{+}^{2}}+\frac{i \bar{\sigma} \cdot D_{-}}{2 a_{L}^{2} x_{-}^{2}}-\frac{m}{a_{R} a_{L} x_{+} x_{-}}, \\
B & =\frac{i \sigma \cdot D_{+}}{2 a_{R}^{2} x_{+}^{2}}-\frac{i \bar{\sigma} \cdot D_{-}}{2 a_{L}^{2} x_{-}^{2}}-\frac{\mu}{a_{R} a_{L} x_{+} x_{-}}, \\
C & =\frac{i \sigma \cdot D_{+}}{2 a_{R}^{2} x_{+}^{2}}-\frac{i \bar{\sigma} \cdot D_{-}}{2 a_{L}^{2} x_{-}^{2}}+\frac{\mu}{a_{R} a_{L} x_{+} x_{-}}, \\
D & =\frac{i \sigma \cdot D_{+}}{2 a_{R}^{2} x_{+}^{2}}+\frac{i \bar{\sigma} \cdot D_{-}}{2 a_{L}^{2} x_{-}^{2}}+\frac{m}{a_{R} a_{L} x_{+} x_{-}},
\end{aligned}
$$


and the two-component spinors $\psi_{+}$and $\psi_{-}$are given by

$$
\psi_{ \pm}=\frac{1}{\sqrt{2}}\left(x_{+} \psi_{R}^{\prime} \pm x_{-} \psi_{L}^{\prime}\right) .
$$

The next step is to determine the coefficients $a_{L(R)}$. To make gauge invariance explicit in the limit $\mu \rightarrow+(-) m$, that is, $x_{+(-)} \rightarrow 0$, only the covariant derivative $D_{+(-)}$should remain. A reasonable choice for the field rescaling is

$$
\frac{1}{2 a_{R}^{2} x_{+}^{2}}=\frac{x_{+}^{2}}{a^{2}} \quad \text { and } \quad \frac{1}{2 a_{L}^{2} x_{-}^{2}}=\frac{x_{-}^{2}}{a^{2}},
$$

where $a$ is an overall numerical coefficient. Thus, we have

$$
a_{R}=\frac{a}{\sqrt{2} x_{+}^{2}} \quad \text { and } \quad a_{L}=\frac{a}{\sqrt{2} x_{-}^{2}},
$$

and we obtain

$$
\begin{aligned}
a^{2} \mathcal{L}_{\mathrm{ferm}}=\left(\psi_{+}^{\dagger} \psi_{-}^{\dagger}\right)\left(\begin{array}{l}
x_{+}^{2} i \sigma \cdot D_{+}+x_{-}^{2} i \bar{\sigma} \cdot D_{-} x_{+}^{2} i \sigma \cdot D_{+}-x_{-}^{2} i \bar{\sigma} \cdot D_{-} \\
x_{+}^{2} i \sigma \cdot D_{+}-x_{-}^{2} i \bar{\sigma} \cdot D_{-} x_{+}^{2} i \sigma \cdot D_{+}+x_{-}^{2} i \bar{\sigma} \cdot D_{-}
\end{array}\right)\left(\begin{array}{c}
\psi_{+} \\
\psi_{-}
\end{array}\right) \\
-2 M\left(\psi_{+}^{\dagger} \psi_{-}^{\dagger}\right)\left(\begin{array}{cc}
1 & \mu / m \\
-\mu / m & -1
\end{array}\right)\left(\begin{array}{c}
\psi_{+} \\
\psi_{-}
\end{array}\right) .
\end{aligned}
$$

Note that the mass matrix is not diagonal, even in the mass eigenbasis, because of the anti-Hermitian mass term controlled by $\mu$.

In the limit $\mu \rightarrow \pm m$ the mass term vanishes, and we are left with a massless theory that is invariant under the gauge transformation

$$
A_{\mu} \longrightarrow A_{\mu}-\partial_{\mu} \phi, \quad \psi_{ \pm} \longrightarrow \begin{cases}\exp \left(i g_{+} \phi\right) \psi_{ \pm}, & \mu=+m \\ \exp \left(i g_{-} \phi\right) \psi_{ \pm}, & \mu=-m\end{cases}
$$

Moreover, from eq. (2.29), we have

$$
\psi_{ \pm}= \begin{cases}\psi_{R}^{\prime}, & \mu=+m \\ \pm \psi_{L}^{\prime}, & \mu=-m\end{cases}
$$

and we find that

$$
\mathcal{L}_{\text {ferm }}= \begin{cases}\psi_{R}^{\dagger} i \sigma \cdot D_{+} \psi_{R}, & \mu=+m, \\ \psi_{L}^{\dagger} i \bar{\sigma} \cdot D_{-} \psi_{L}, & \mu=-m,\end{cases}
$$

for massless right- and left-chiral theories, as observed in the preceding subsections.

The coefficients $a_{L(R)}$ and the transformation in eq. (2.26) are singular in the limit $\mu \rightarrow \pm m$. However, the coefficients $a_{L(R)}$ do not appear in the final Lagrangian [eq. (2.32)], which remains finite in the limit $\mu \rightarrow \pm m$. Furthermore, the functional Jacobian of the field rescaling in eq. (2.24), although also singular, cancels out in the normalization of $Z$ with the partition function $Z_{0}$ of the corresponding free theory. Thus, the limit $\mu \rightarrow \pm m$ may be taken safely, as done above. 


\subsection{Exceptional points}

An $N$-dimensional Hermitian matrix always has $N$ real eigenvalues and associated with each eigenvalue is a distinct eigenvector. For non-Hermitian matrices the situation is more elaborate. Consider, for example, the non-Hermitian 2-dimensional matrix

$$
A=\left(\begin{array}{cc}
a+i b & g \\
g & a-i b
\end{array}\right),
$$

where $a$ and $b$ are real parameters and $g$ is a real coupling constant. The eigenvalues of $A$ are $E(g)=a \pm \sqrt{g^{2}-b^{2}}$. Thus, there are two phases: a broken phase (for $g^{2}<b^{2}$ ) in which the eigenvalues are complex and an unbroken phase (for $g^{2}>b^{2}$ ) in which the eigenvalues are real. At the boundary between the phases $(g= \pm b)$ the eigenvalues merge, and the matrix is said to be defective because there is only one eigenvalue $E=a$ and one eigenvector instead of two: $(i, 1)$ for $g=+b$ and $(1, i)$ for $g=-b$. The point $g^{2}=b^{2}$ is called an exceptional point.

In general, at an exceptional point a pair of eigenvalues of a non-Hermitian matrix merge, and one of the eigenvectors disappears. (It is possible for more than two eigenvalues to merge at an exceptional point, but this is not common.) If the exceptional point occurs when a parameter $g$ has the value $g_{0}$, the eigenvalues $E(g)$ exhibit a square-root singularity at $g=g_{0}$.

Hermitian matrices do not have exceptional points. Nevertheless, exceptional points play a crucial role in explaining their behavior. For example, in conventional Hermitian quantum theory the radius of convergence of a perturbation expansion in powers of a coupling constant is precisely the distance to the nearest exceptional point (a square-root singularity) in the complex-coupling-constant plane [32].

In the limit $\mu= \pm m$ the mass matrix $M$ is defective and, as explained above, the transformation in eq. (2.26) becomes singular. For instance, for $\mu=+m$ the mass matrix has the Jordan normal form

$$
\boldsymbol{M}=2 m\left(\begin{array}{ll}
0 & 1 \\
0 & 0
\end{array}\right),
$$

with zero eigenvalues. In this limit we have chiral flips biased from left to right, depleting the probability density of the left-handed component, as we saw in eq. (2.10). In other words, we again arrive at a massless theory of right-handed fields. Conversely, in the limit $\mu \rightarrow-m$ we arrive at a massless theory dominated by left-handed fields with chiral flips biased from right to left.

The appearance of defective matrices is rare in physics, especially in field theory, and it is worth considering what this singular behaviour signals. For $\mu^{2}<m^{2}$ we have rightand left-chiral components with positive mass-squared $M^{2}>0$ (time-like) and real-valued energies $\omega \in \mathbb{R}$. For $\mu>0$ the right-chiral component dominates; for $\mu<0$ the left-chiral component dominates; for $\mu=0$ we have exact symmetry between both components. On the other hand, for $\mu^{2}>m^{2}$ we still have right- and left-chiral components, but these are now tachyonic, having negative mass-squared $M^{2}<0$ (space-like) and imaginary-valued 


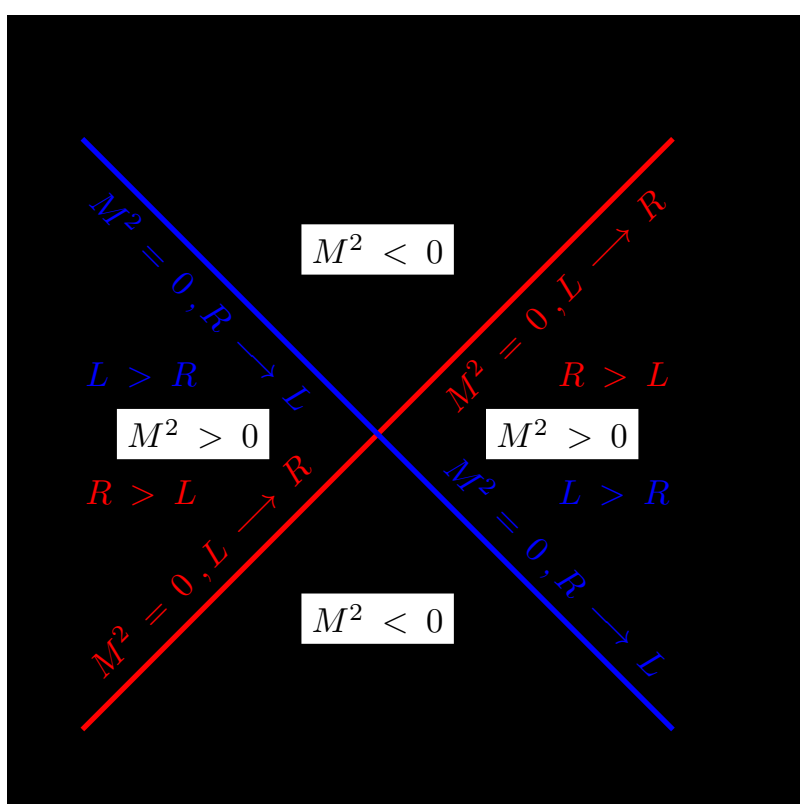

Figure 1. Schematic representation of the $m-\mu$ plane, where the tachyonic region (cross-hatched) is bounded by the lines $\mu^{2}=m^{2}$, along which the mass matrix becomes defective: $\mu=+m$ (red) corresponds to total left-chiral domination, and $\mu=-m$ (blue) corresponds to total right-chiral domination. Along the line $\mu=0$ the symmetry between the right- and left-chiral components is restored.

energies $i \omega \in \mathbb{R}$. For the special case $\mu^{2}=m^{2}$ we have a massless fermion $M^{2}=0$ (lightlike) and real-valued energies $\omega=|\vec{p}| \in \mathbb{R}$. For $\mu=+m$ this field is completely dominated by its right-chiral component and for $\mu=-m$ it is completely dominated by its left-chiral component. The mass matrix becomes defective at the boundary between the time-like particle and space-like tachyonic regimes. This is indicated graphically in figure 1.

\section{One-loop corrections}

We give here the one-loop corrections to the fermion and photon self-energies, as well as the three-point vertex. The technical details of the calculations are given explicitly in appendix A. For our purposes it is convenient to express the one-loop results in terms of the Passarino-Veltman form factors [33], the definitions of which are also given in appendix A. We work in the Feynman gauge throughout, with the gauge-fixing term

$$
\mathcal{L}_{\text {gf }}=\frac{1}{2}\left(\partial_{\mu} A^{\mu}\right)^{2}
$$

in which the photon propagator has the simple form

$$
i D_{\mu \nu}(k)=\frac{i \eta_{\mu \nu}}{k^{2}+i \varepsilon} .
$$

Fermion self-energy. There are four contributions to the one-loop fermion self-energy: one with two vector couplings, one with two axial couplings, and two with one vector 
and one axial coupling. Employing dimensional regularization and working in $d=4-2 \epsilon$ dimensions, we find the total self-energy

$$
\Sigma(p)=\frac{2-d}{16 \pi^{2}}\left(g_{V}-g_{A} \gamma^{5}\right)^{2} \not p B_{1}+\frac{d}{16 \pi^{2}}\left(g_{V}^{2}-g_{A}^{2}\right)\left(m+\mu \gamma^{5}\right) B_{0},
$$

where we have suppressed the arguments on the form factors $B_{0 ; 1} \equiv B_{0 ; 1}(p, M, 0)$. Isolating the logarithmically-divergent part, we obtain

$$
\Sigma(p)=\frac{1}{16 \pi^{2} \epsilon}\left(\not p\left(g_{V}+g_{A} \gamma^{5}\right)^{2}+4\left(g_{V}^{2}-g_{A}^{2}\right)\left(m+\mu \gamma^{5}\right)\right)+\text { finite },
$$

where higher orders in $p$ are omitted.

The RL and LR components of the fermion self-energy are given by

$$
\begin{aligned}
& \Sigma_{R L}(p)=\frac{g_{+} g_{-}}{16 \pi^{2}} d m_{-} B_{0}, \\
& \Sigma_{L R}(p)=\frac{g_{+} g_{-}}{16 \pi^{2}} d m_{+} B_{0} .
\end{aligned}
$$

For $\mu= \pm m$ we see that one of these components vanishes such that it remains the case that only the operator $\psi_{L}^{\dagger} \psi_{R}(\mu=+m)$ or $\psi_{R}^{\dagger} \psi_{L}(\mu=-m)$ is present, preserving the argument in subsection 2.2. Specifically, the equations of motion for the renormalized rightand left-chiral fields at order $g^{2}$ are given by

$$
\begin{aligned}
Z_{R} i \sigma^{\mu} \cdot D_{+} \psi_{R} & =0, \\
Z_{L} i \bar{\sigma}^{\mu} \cdot D_{-} \psi_{L} & =\left(2 m+\delta m-\Sigma_{L R}(p)\right) \psi_{R},
\end{aligned}
$$

where $\delta m$ is the mass counterterm and in the on-shell scheme the wavefunction renormalization $Z_{R(L)}$ is given by

$$
Z_{R(L)}=1-\left.\frac{\mathrm{d}}{\mathrm{d} \not p} \Sigma_{R R(L L)}(p)\right|_{p^{2}=0}
$$

We may again integrate out the left-chiral component, obtaining a massless right-handed theory also at order $g^{2}$.

Polarization tensor. There are also four contributions to the one-loop photon polarization tensor, and we find the total polarization tensor

$$
\Pi^{\mu \nu}(p)=-\frac{g_{V}^{2}+g_{A}^{2}}{2 \pi^{2}}\left(p^{\mu} p^{\nu}-\eta^{\mu \nu} p^{2}\right)\left(B_{21}+B_{1}\right)+\frac{g_{A}^{2}}{\pi^{2}} \eta^{\mu \nu} M^{2} B_{0} .
$$

The form factors are evaluated as $B_{0 ; 1 ; 21} \equiv B_{0 ; 1 ; 21}(p, M, M)$. As expected from the loss of gauge invariance in the case of axially-coupled massive fermions, the polarization tensor is not transverse and contains the longitudinal part

$$
\Pi^{\mu \nu}(p) \supset \frac{g_{A}^{2}}{\pi^{2}} \eta^{\mu \nu} M^{2} B_{0} .
$$

Nevertheless, this longitudinal part vanishes and the polarization tensor becomes transverse when $\mu^{2}=m^{2}$ (that is, when $M=0$ ). Hence, as a consequence of the restoration of 
gauge invariance (see subsection 2.3), the polarization tensor satisfies the standard QED Ward identity.

Isolating the logarithmic divergences in eq. (3.8), we obtain

$$
\Pi^{\mu \nu}(p)=\frac{g_{V}^{2}+g_{A}^{2}}{12 \pi^{2} \epsilon}\left(p^{\mu} p^{\nu}-\eta^{\mu \nu} p^{2}\right)+\frac{g_{A}^{2}}{\pi^{2} \epsilon} M^{2} \eta^{\mu \nu}+\text { finite },
$$

where higher orders in $p$ are omitted.

Vertex. The four different contributions to the three-point vertex lead to the total oneloop correction

$$
\begin{aligned}
\Lambda^{\mu}(p, q)= & \frac{2-d}{16 \pi^{2}}\left(g_{V}+g_{A} \gamma^{5}\right) \\
& \times\left\{\left(g_{V}+g_{A} \gamma^{5}\right)^{2}\left[(2-d) \gamma^{\mu} C_{24}+\gamma^{\rho} \gamma^{\mu} \gamma^{\kappa} F_{\kappa \rho}\right]+\left(g_{V}^{2}+g_{A}^{2}\right) \gamma^{\mu} M^{2} C_{0}\right\} \\
& +\frac{1}{4 \pi^{2}}\left(g_{V}^{2}+g_{A}^{2}\right)\left(m-\mu \gamma^{5}\right) \\
& \times\left\{g_{V}\left[p^{\mu}\left(2 C_{11}+C_{0}\right)+q^{\mu}\left(2 C_{12}+C_{0}\right)\right]-g_{A} \gamma^{5}\left(p^{\mu}+q^{\mu}\right) C_{0}\right\},
\end{aligned}
$$

where we have defined

$$
F_{\kappa \rho}=p_{\kappa} p_{\rho}\left(C_{11}+C_{21}\right)+q_{\kappa} q_{\rho}\left(C_{22}+C_{12}\right)+p_{\kappa} q_{\rho}\left(C_{23}+C_{11}\right)+q_{\kappa} p_{\rho}\left(C_{23}+C_{12}\right) .
$$

The three-point form factors (see appendix $\mathrm{A}$ ) are evaluated with arguments $p_{1}=p, p_{2}=q$, $m_{1}=m_{3}=M$, and $m_{2}=0$. The divergent contribution to eq. (3.11) arises from the form factor $C_{24}$ and is given by

$$
\Lambda^{\mu} \supset \frac{1}{16 \pi^{2} \epsilon}\left(g_{V}+g_{A} \gamma^{5}\right)^{3} \gamma^{\mu}
$$

which is consistent with the self-energy [eq. (3.4)], as imposed by the Ward identity for $g_{A} \rightarrow 0$, describing the usual gauge invariance of vectorially-coupled massive QED.

Finally, the RL and LR components of the vertex, that is, those mediating right-to-left and left-to-right chiral flips, are given by

$$
\begin{aligned}
& \Lambda_{R L}^{\mu}=\frac{g_{+} g_{-}}{4 \pi^{2}} m_{-}\left[\left(g_{+}+g_{-}\right)\left(p^{\mu} C_{11}+q^{\mu} C_{12}\right)+g_{-}\left(p^{\mu}+q^{\mu}\right) C_{0}\right], \\
& \Lambda_{L R}^{\mu}=\frac{g_{+} g_{-}}{4 \pi^{2}} m_{+}\left[\left(g_{+}+g_{-}\right)\left(p^{\mu} C_{11}+q^{\mu} C_{12}\right)+g_{+}\left(p^{\mu}+q^{\mu}\right) C_{0}\right] .
\end{aligned}
$$

Like the RL and LR components of the fermion self-energies, these terms are proportional to $m_{-}$and $m_{+}$, respectively, so we have only left-to-right chiral flips for $\mu=+m$ and rightto-left chiral flips for $\mu=-m$, which preserves the structure observed in subsection 2.2 for $\mu^{2}=m^{2}$ also at order $g^{3}$.

\section{Implications for neutrino masses}

This section highlights potential implications of the behavior of this non-Hermitian theory for the neutrino sector of the SM. We extend the SM with a right-handed singlet neutrino $\nu_{R}$. In the Dirac basis we write the non-Hermitian neutrino Yukawa sector, assuming only a single generation for now, as

$$
\mathcal{L}=\bar{L}_{L} i \not D L_{L}+\bar{\nu}_{R} i \not \nu_{R}-h_{-} \bar{L}_{L} \widetilde{\phi} \nu_{R}-h_{+} \bar{\nu}_{R} \widetilde{\phi}^{\dagger} L_{L}
$$


where $L_{L}=\left(\nu_{L}, e_{L}\right)$ is the $\mathrm{SU}(2)$ lepton doublet, $\widetilde{\phi}=i \sigma_{2} \phi^{*}$ is the isospin conjugate of the Higgs doublet and $D_{\mu}$ is the usual covariant derivative of the SM gauge groups. Note that we have swapped + and - relative to the non-Hermitian model of QED in the preceding sections. The non-Hermitian Yukawa couplings are

$$
h_{ \pm}=h \pm \eta
$$

where, for now, we assume that $h, \eta \in \mathbb{R}$. Since the electroweak sector of the SM is already written in terms of chiral fields, no $\gamma^{5}$ appears explicitly in the non-Hermitian Lagrangian. Even so, in the symmetry-broken phase, the non-Hermitian Yukawa couplings give rise to a Hermitian mass $m=v h$ and an anti-Hermitian mass $\mu=v \eta$, with $m_{ \pm}=v(h \pm \eta)$, in complete analogy to the non-Hermitian Abelian theory considered in section 2.

In the unitary gauge and after spontaneous symmetry breaking, the Higgs doublet takes the form

$$
\phi=\frac{1}{\sqrt{2}}\left(\begin{array}{c}
0 \\
v+H
\end{array}\right), \quad \widetilde{\phi}=\frac{1}{\sqrt{2}}\left(\begin{array}{c}
v+H \\
0
\end{array}\right) .
$$

Hence, the neutrino sector becomes

$$
\mathcal{L}_{\nu}=\bar{\nu}_{L} i \not \partial \nu_{L}+\bar{\nu}_{R} i \not \nu_{R}-h_{-} \frac{v}{\sqrt{2}} \bar{\nu}_{L} \nu_{R}-h_{+} \frac{v}{\sqrt{2}} \bar{\nu}_{R} \nu_{L}-\frac{h_{-}}{\sqrt{2}} \bar{\nu}_{L} H \nu_{R}-\frac{h_{+}}{\sqrt{2}} \bar{\nu}_{R} H \nu_{L}
$$

The first four terms of the Lagrangian in eq. (4.4) can be written in the matrix form

$$
\mathcal{L}_{\nu} \supset\left(\bar{\nu}_{\mathrm{L}} \bar{\nu}_{\mathrm{R}}\right)\left(\begin{array}{cc}
i \not \partial & -h_{-} \frac{v}{\sqrt{2}} \\
-h_{+} \frac{v}{\sqrt{2}} & i \not \partial
\end{array}\right)\left(\begin{array}{c}
\nu_{\mathrm{L}} \\
\nu_{\mathrm{R}}
\end{array}\right),
$$

where the neutrino mass matrix

$$
\boldsymbol{M}=\frac{v}{\sqrt{2}}\left(\begin{array}{cc}
0 & h_{-} \\
h_{+} & 0
\end{array}\right)
$$

has eigenvalues

$$
\pm M= \pm \frac{v}{\sqrt{2}} \sqrt{h^{2}-\eta^{2}}
$$

Proceeding in analogy to subsection 2.3, we make the field redefinition $(a=\sqrt{2})$

$$
\nu_{L(R)} \longrightarrow \nu_{L(R)}^{\prime}=\frac{\nu_{L(R)}}{x_{-(+)}^{2}},
$$

where

$$
x_{ \pm} \equiv \sqrt{1 \pm \eta / h}
$$

We then move to the mass eigenbasis spanned by the two-component spinors

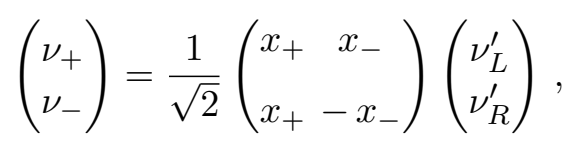


with

$$
\nu_{ \pm}=\frac{1}{\sqrt{2}}\left(x_{+} \nu_{L}^{\prime} \pm x_{-} \nu_{R}^{\prime}\right) .
$$

Thus, in the limit $\eta \rightarrow h$ and in analogy to subsection 2.3 , we obtain a theory of massless left-handed neutrinos, which is the "original" Standard Model. However, arranging for $\eta \sim h$ with $\eta<0$, we obtain a nonzero but arbitrarily small mass for the neutrinos, with the propagating state still dominated by its left-chiral component.

In the above minimal extension of the SM the singlet neutrino $\nu_{R}$ does not couple to the $\mathrm{SU}(2)_{L}$ gauge fields, and we cannot make use of an analogy to the non-Hermitian Abelian gauge couplings of subsection 2.1. However, in the so-called left-right SM [34-36], where the $\mathrm{SM}$ gauge groups are extended from $\mathrm{SU}(2)_{L} \otimes \mathrm{U}(1)_{Y}$ to $\mathrm{SU}(2)_{L} \otimes \mathrm{SU}(2)_{R} \otimes \mathrm{U}(1)_{B-L}$, the $\mathrm{SU}(2)_{R}$ gauge fields couple directly to the right-handed neutrino current. Thus, by introducing couplings $g_{+}=g_{V}+g_{A}$ and $g_{-}=g_{V}-g_{A}$ of the left- and right-handed currents to the charged gauge fields $W_{L}^{ \pm, \mu}$ and $W_{R}^{ \pm, \mu}$, and $g_{+}^{\prime}=g_{V}^{\prime}+g_{A}^{\prime}$ and $g_{-}^{\prime}=g_{V}^{\prime}-g_{A}^{\prime}$ to the neutral gauge fields $Z_{L}^{\mu}$ and $Z_{R}^{\mu}$, those of the right-handed neutrino may be suppressed for $g_{V}{ }^{(\prime)} \sim g_{A}{ }^{(\prime)}$. This, of course, amounts only to choosing different values for the tree-level $\mathrm{SU}(2)_{L}$ and $\mathrm{SU}(2)_{R}$ gauge couplings, which need not result from a non-Hermitian theory. Nevertheless, this construction might provide a common origin for such a structure in the gauge and Yukawa sectors.

The masses of the left- and right-handed neutrinos are degenerate in this construction, both being light for $\eta \sim h$. However, since the right-handed neutrino is still a singlet of the SM gauge groups, we are not precluded from adding a Majorana mass term

$$
\mathcal{L}_{\nu} \supset-m_{R} \bar{\nu}_{R}^{\mathcal{C}} \nu_{R}
$$

where $\mathcal{C}$ denotes charge conjugation. In this case, the Lagrangian takes the form

$$
-\mathcal{L}_{\nu} \supset \frac{1}{2}\left(\bar{\nu}_{L} \bar{\nu}_{R}^{\mathcal{C}}\right)\left(\begin{array}{cc}
0 & m_{-} \\
m_{+} & m_{R}
\end{array}\right)\left(\begin{array}{c}
\nu_{L}^{\mathcal{C}} \\
\nu_{R}
\end{array}\right)+\frac{1}{2}\left(\begin{array}{ll}
\bar{\nu}_{L}^{\mathcal{C}} & \bar{\nu}_{R}
\end{array}\right)\left(\begin{array}{cc}
0 & m_{-} \\
m_{+} & m_{R}
\end{array}\right)\left(\begin{array}{c}
\nu_{L} \\
\nu_{R}^{\mathcal{C}}
\end{array}\right) .
$$

For $m_{R} \gg 2 M$ the masses of the light and heavy neutrinos are $m_{L}=-M^{2} / m_{R}$ and $m_{R}$, which drives up the mass of the right-handed neutrino and further suppresses that of the left-handed neutrino by means of the see-saw mechanism [24-28].

It is worth commenting on the generalization to complex Hermitian and anti-Hermitian Yukawa couplings $h$ and $\eta$. In this case, the Yukawa sector takes the form

$$
\mathcal{L}_{\nu} \supset-h_{-} \frac{v}{\sqrt{2}} \bar{\nu}_{L} \nu_{R}-h_{+}^{*} \frac{v}{\sqrt{2}} \bar{\nu}_{R} \nu_{L} .
$$

The mass-squared is then given by

$$
M^{2}=\frac{v^{2}}{4}\left(|h|^{2}-|\eta|^{2}-2 i \operatorname{Im} h^{*} \eta\right),
$$

which delivers real masses only when $\operatorname{Im} h^{*} \eta$ vanishes, i.e. when $h=\eta$. Thus, if we want small but finite masses, we are required to take $h$ and $\eta$ to be real. 
The situation is somewhat different, however, when we consider the extension of the above model to include $N$ generations:

$$
\mathcal{L}=\bar{L}_{L}^{k} i \not D L_{L, k}+\bar{\nu}_{R}^{\alpha} i \not \nu_{R, \alpha}-\left[h_{-}\right]_{k}^{\alpha} \bar{L}_{L}^{k} \widetilde{\phi} \nu_{\mathrm{R}, \alpha}-\left[h_{+}\right]_{\alpha}^{k} \bar{\nu}_{R}^{\alpha} \widetilde{\phi}^{\dagger} L_{L, k}
$$

where we have assumed only Dirac masses in the first instance. We have employed the flavour-covariant notation of ref. [37], where the left- and right-handed sectors transform in the fundamental representation of two flavour groups $U_{L}(N)$ and $U_{R}(N)$, respectively, and flavour indices are raised and lowered by complex conjugation. We have taken the number of left- and right-handed fields to be equal for simplicity in what follows; this need not be the case in general. Under a general transformation in $U_{L}(N) \times U_{R}(N)$, we have

$$
\begin{aligned}
L_{L, k} \longrightarrow L_{L, k}^{\prime}=V_{k}{ }^{l} L_{L, l}, & L_{L}^{k} \equiv\left(L_{L, k}\right)^{\dagger} \longrightarrow L_{L}^{\prime k}=V^{k}{ }_{l} L_{L}^{l}, \\
\nu_{R, \alpha} \longrightarrow \nu_{R, \alpha}^{\prime}=U_{\alpha}{ }^{\beta} \nu_{R, \beta}, & \nu_{R}^{\alpha} \equiv\left(\nu_{R, \alpha}\right)^{\dagger} \longrightarrow \nu_{R}^{\prime \alpha}=U^{\alpha}{ }_{\beta} \nu_{R}^{\beta},
\end{aligned}
$$

where $V_{l}^{k} \equiv\left(V_{k}{ }^{l}\right)^{*} \in U_{L}(N)$ and $U_{\beta}^{\alpha} \equiv\left(U_{\alpha}{ }^{\beta}\right)^{*} \in U_{R}(N)$. The Yukawa coupling matrices $\boldsymbol{h}_{ \pm}=\boldsymbol{h} \pm \boldsymbol{\eta}$ transform as tensors of $U_{L}(N) \times U_{R}(N)$ and flavour covariance of the Lagrangian requires the transformation property

$$
\left[h_{ \pm}\right]_{k}^{\alpha} \longrightarrow\left[h_{ \pm}^{\prime}\right]_{k}^{\alpha}=V_{k}^{l} U_{\beta}^{\alpha}\left[h_{ \pm}\right]_{l}^{\beta}
$$

In general, there will not exist a flavour basis in which the Yukawa matrices $\boldsymbol{h}_{+}$and $\boldsymbol{h}_{-}$are simultaneously diagonal. As a result, there can be a three-fold misalignment for general Yukawa matrices, i.e. the weak, + Yukawa and - Yukawa bases can point in three different directions in flavour space. Hence, for three generations, neutrino oscillations in this model are governed by 6 rather than 3 mixing angles and 2 rather than $1 \mathcal{C} \mathcal{P}$-violating phases. This additional source of $\mathcal{C P}$ violation is of particular relevance to the potential embedding of this non-Hermitian theory within the scenario of leptogenesis.

In the symmetry-broken phase, for the case of two generations $(N=2)$, the mass spectrum contains four mass eigenstates with masses given by the roots of

$$
M_{1(2)}^{2}=\frac{v^{2}}{4}\left[\operatorname{tr} \boldsymbol{h}_{+}^{\dagger} \boldsymbol{h}_{-}-(+)\left(2 \operatorname{tr}\left(\boldsymbol{h}_{+}^{\dagger} \boldsymbol{h}_{-}\right)^{2}-\left(\operatorname{tr} \boldsymbol{h}_{+}^{\dagger} \boldsymbol{h}_{-}\right)^{2}\right)^{1 / 2}\right]
$$

It is clear that one may obtain the massless limit by choosing $\boldsymbol{h}= \pm \boldsymbol{\eta}$. However, such a constraint is not a necessary condition for obtaining a spectrum with massless states. In the case that

$$
\operatorname{det} \boldsymbol{h}_{+}^{\dagger} \boldsymbol{h}_{-}=0 \quad \Rightarrow \quad \operatorname{tr}\left(\boldsymbol{h}_{+}^{\dagger} \boldsymbol{h}_{-}\right)^{2}=\left(\operatorname{tr} \boldsymbol{h}_{+}^{\dagger} \boldsymbol{h}_{-}\right)^{2}
$$

we obtain two massless states $\left( \pm M_{1}=0\right)$ and two states with masses given by the roots of

$$
M_{2}^{2}=\frac{v^{2}}{2} \operatorname{tr} \boldsymbol{h}_{+}^{\dagger} \boldsymbol{h}_{-}=\frac{v^{2}}{2}\left[\operatorname{tr} \boldsymbol{h}^{\dagger} \boldsymbol{h}-\operatorname{tr} \boldsymbol{\eta}^{\dagger} \boldsymbol{\eta}-2 i \operatorname{Im} \operatorname{tr} \boldsymbol{h}^{\dagger} \boldsymbol{\eta}\right]
$$

For $M_{2}$ to be real, we require

$$
\operatorname{Im} \operatorname{tr} \boldsymbol{h}^{\dagger} \boldsymbol{\eta}=0
$$


Subsequently imposing the additional constraint that

$$
\operatorname{tr} \boldsymbol{h}^{\dagger} \boldsymbol{h}=\operatorname{tr} \boldsymbol{\eta}^{\dagger} \boldsymbol{\eta}
$$

we also obtain $M_{2}=0$, giving a massless spectrum. In complete analogy to the singleflavour case, we may obtain an arbitrarily small but finite mass splitting $\Delta M^{2}=M_{2}^{2}-M_{1}^{2}$ by choosing

$$
\operatorname{tr} \boldsymbol{h}^{\dagger} \boldsymbol{h} \sim \operatorname{tr} \boldsymbol{\eta}^{\dagger} \boldsymbol{\eta}
$$

As a result, there is the potential to obtain sub-eV scale Dirac neutrino masses from Hermitian and anti-Hermitian Yukawa couplings, whose orders of magnitude may themselves be much closer to the other SM Yukawa couplings and larger than the unnatural $10^{-12}$ that would otherwise be required for agreement with neutrino oscillation data.

Note that eqs. (4.20), (4.22) and (4.23) comprise three constraints on the total of 16 parameters in the complex-valued $2 \times 2$ matrices $\boldsymbol{h}$ and $\boldsymbol{\eta}$. These three necessary conditions provide a much weaker constraint on the elements of $\boldsymbol{h}$ and $\boldsymbol{\eta}$ than the condition $\boldsymbol{h}=\boldsymbol{\eta}$. Moreover, they do not, as in the single-flavour case, require $\boldsymbol{h}$ and $\boldsymbol{\eta}$ to be real-valued matrices.

As for the single-flavour case, we can include a Majorana mass term of the form

$$
-\mathcal{L}_{\nu} \supset \frac{1}{2} \bar{\nu}_{R, \alpha}^{\mathcal{C}} m_{R}^{\alpha \beta} \nu_{R, \beta}+\text { H.c. },
$$

where the mass matrix $\boldsymbol{m}_{R}$ transforms as a rank-2 tensor of $U_{R}(N)$, i.e.

$$
m_{R}^{\alpha \beta} \longrightarrow m_{R}^{\prime \alpha \beta}=U_{\gamma}^{\alpha} U^{\beta}{ }_{\delta} m_{R}^{\gamma \delta} .
$$

In block form the mass terms are given by

$$
\begin{aligned}
-\mathcal{L}_{\nu} \supset \frac{1}{2}\left(\bar{\nu}_{L}^{k} \bar{\nu}_{R, \alpha}^{\mathcal{C}}\right)\left(\begin{array}{cc}
0 & {\left[m_{-}\right]_{k}^{\beta}} \\
{\left[m_{+}\right]_{l}^{\alpha}} & m_{R}^{\alpha \beta}
\end{array}\right)\left(\begin{array}{c}
\nu_{L}^{\mathcal{C}, l} \\
\nu_{R, \beta}
\end{array}\right) \\
+\frac{1}{2}\left(\begin{array}{cc}
\bar{\nu}_{L, k}^{\mathcal{C}} & \bar{\nu}_{R}^{\alpha}
\end{array}\right)\left(\begin{array}{cc}
0 & {\left[m_{-}\right]_{\beta}^{k}} \\
{\left[m_{+}\right]_{\alpha}^{l}} & m_{R, \alpha \beta}
\end{array}\right)\left(\begin{array}{c}
\nu_{L, l} \\
\nu_{R}^{\mathcal{C}, \beta}
\end{array}\right) .
\end{aligned}
$$

The mass matrix

$$
M=\left(\begin{array}{cc}
\mathbf{0} & \boldsymbol{m}_{-} \\
\boldsymbol{m}_{+}^{\top} & \boldsymbol{m}_{R}
\end{array}\right)
$$

can be block diagonalized by a unitary transformation of the form $\widehat{\boldsymbol{M}}=\boldsymbol{W}^{\top} \boldsymbol{M W}$, giving the physical neutrinos

$$
\left(\begin{array}{c}
N_{L} \\
N_{R}^{\mathcal{C}}
\end{array}\right)=\boldsymbol{W}^{\top}\left(\begin{array}{c}
\nu_{L} \\
\nu_{R}^{\mathcal{C}}
\end{array}\right), \quad\left(\begin{array}{c}
N_{L}^{\mathcal{C}} \\
N_{R}
\end{array}\right)=\boldsymbol{W}^{\dagger}\left(\begin{array}{c}
\nu_{L}^{\mathcal{C}} \\
\nu_{R}
\end{array}\right),
$$

where the $N_{L}$ are the light neutrinos, whose mass matrix is given by the non-Hermitian see-saw formula

$$
\boldsymbol{m}_{L}=-\boldsymbol{m}_{-} \boldsymbol{m}_{R}^{-1} \boldsymbol{m}_{+}^{\top},
$$

and the $N_{R}$ are the heavy Majorana neutrinos, whose mass matrix is $\boldsymbol{m}_{R}$. 
For $N=2$ the mass spectrum of the light neutrinos is given by

$$
M_{1(2)}=-\frac{v^{2}}{4}\left[\operatorname{tr} \boldsymbol{h}_{-} \boldsymbol{m}_{R}^{-1} \boldsymbol{h}_{+}^{\top}-(+)\left(2 \operatorname{tr}\left(\boldsymbol{h}_{-} \boldsymbol{m}_{R}^{-1} \boldsymbol{h}_{+}^{\top}\right)^{2}-\left(\operatorname{tr} \boldsymbol{h}_{-} \boldsymbol{m}_{R}^{-1} \boldsymbol{h}_{+}^{\top}\right)^{2}\right)^{1 / 2}\right] .
$$

We trivially obtain a massless spectrum for $\boldsymbol{h}= \pm \boldsymbol{\eta}$. However, as before, when

$$
\operatorname{det} \boldsymbol{h}_{-} \boldsymbol{m}_{R}^{-1} \boldsymbol{h}_{+}^{\top}=0 \quad \Rightarrow \quad \operatorname{tr}\left(\boldsymbol{h}_{-} \boldsymbol{m}_{R}^{-1} \boldsymbol{h}_{+}^{\top}\right)^{2}=\left(\operatorname{tr} \boldsymbol{h}_{-} \boldsymbol{m}_{R}^{-1} \boldsymbol{h}_{+}^{\top}\right)^{2},
$$

we obtain the spectrum

$$
M_{1}=0, \quad M_{2}=-\frac{v^{2}}{2} \operatorname{tr} \boldsymbol{h}_{-} \boldsymbol{m}_{R}^{-1} \boldsymbol{h}_{+}^{\top} .
$$

For $M_{2}$ to be real, we now require

$$
\operatorname{Im} \operatorname{tr} \boldsymbol{h}_{-} \boldsymbol{m}_{R}^{-1} \boldsymbol{h}_{+}^{\top}=0,
$$

and we obtain a completely massless spectrum if, in addition, we require that

$$
\operatorname{Re} \operatorname{tr} \boldsymbol{h}_{-} \boldsymbol{m}_{R}^{-1} \boldsymbol{h}_{+}^{\top}=0 .
$$

Again, the conditions eqs. (4.32), (4.34) and (4.35) provide much weaker constraints on the form of the Yukawa matrices than $\boldsymbol{h}= \pm \boldsymbol{\eta}$. In addition, we can obtain an arbitrarily small but finite mass splitting $\Delta M^{2}$, independent of the Majorana mass term $\boldsymbol{m}_{R}$, by choosing the Yukawa couplings such that

$$
\operatorname{Retr} \boldsymbol{h} \boldsymbol{m}_{R}^{-1} \boldsymbol{h}^{\top} \sim \operatorname{Retr}\left(\boldsymbol{\eta} \boldsymbol{m}_{R}^{-1} \boldsymbol{\eta}^{\top}+\boldsymbol{h} \boldsymbol{m}_{R}^{-1} \boldsymbol{\eta}^{\top}-\boldsymbol{\eta} \boldsymbol{m}_{R}^{-1} \boldsymbol{h}^{\top}\right) .
$$

This ability to tune the mass splitting of the light neutrinos independent of the magnitude of the Majorana mass term may have interesting implications in the light of the combined constraints provided by neutrino oscillation data and the current limits on leptonflavour-violating and lepton-number-violating observables, including neutrinoless doublebeta decay.

A comprehensive phenomenological study of the aforementioned variations of this nonHermitian Yukawa model in the context of current constraints from collider experiments and both astrophysical and cosmological observations (for recent reviews, see refs. [38, 39]) is beyond the scope of this article and will be presented elsewhere.

\section{Conclusions}

We have considered an extension of QED, whose non-Hermitian nature permits the symmetry between the left- and right-chiral components of a Dirac fermion to be broken by the presence of an anti-Hermitian mass term. We have shown that the full gauge invariance of this theory is restored when the Hermitian and anti-Hermitian masses are of equal magnitude. Moreover, we have highlighted an intriguing possibility for explaining the smallness of the light neutrino masses and providing an additional source of $\mathcal{C P}$ violation through an analogous extension of the SM. Further phenomenological studies of this model and its 
variations are required in the context of the current low-energy neutrino data as well as both cosmological and astrophysical observations.

Finally, we mention another direction of study, which deals with the dynamical generation of the non-Hermitian mass term through nonperturbative quantum effects. Dynamical mass generation for neutrinos (with a vanishing bare mass) has been obtained in the context of Lorentz-symmetry violation [40-42], where the physical mass scale is provided by higher-order spatial derivatives. A nonperturbative mechanism could also be responsible for the non-Hermitian mass term in the present context, although the natural mass scale would be provided by the Higgs mechanism, instead of Lorentz-symmetry-violating operators. In order to explore this avenue, one needs to derive a nonperturbative gap equation and study the possibility of a non-Hermitian mass term solution. Such a nontrivial solution could arise in a theory involving an axial coupling and is left for future work.

\section{Acknowledgments}

The work of P.M. is supported by a University Foundation Fellowship (TUFF) from the Technische Universität München and by the Deutsche Forschungsgemeinschaft cluster of excellence Origin and Structure of the Universe.

\section{A One-loop corrections}

This appendix summarizes the technical details of the one-loop calculations described in section 3. The elements $A_{I J}$ of a matrix $\boldsymbol{A}$ in the chiral field space are indexed by upper-case Roman indices $I, J, K, M, \cdots=L, R$, where the LL element is in the top left.

Passarino-Veltman parametrization. In $d=4-2 \epsilon$ the two-point Passarino-Veltman form factors [33] are

$$
B_{0 ; \mu ; \mu \nu}\left(p, m_{1}, m_{2}\right)=\int \frac{\mathrm{d}^{d} k}{i \pi^{2}} \frac{1 ; k_{\mu} ; k_{\mu} k_{\nu}}{\left(k^{2}-m_{1}^{2}+i \varepsilon\right)\left((p+k)^{2}-m_{2}^{2}+i \varepsilon\right)} .
$$

These may be related to the scalar form factors $B_{1}, B_{21}$ and $B_{22}$ via

$$
\begin{aligned}
B_{\mu}\left(p, m_{1}, m_{2}\right) & =p_{\mu} B_{1}\left(p, m_{1}, m_{2}\right), \\
B_{\mu \nu}\left(p, m_{1}, m_{2}\right) & =p_{\mu} p_{\nu} B_{21}\left(p, m_{1}, m_{2}\right)+\eta_{\mu \nu} B_{22}\left(p, m_{1}, m_{2}\right),
\end{aligned}
$$

whose divergent parts are

$$
\begin{aligned}
& B_{0}\left(p, m_{1}, m_{2}\right) \supset \frac{1}{\epsilon}, \quad B_{1}\left(p, m_{1}, m_{2}\right) \supset-\frac{1}{2 \epsilon}, \\
& B_{21}\left(p, m_{1}, m_{2}\right) \supset \frac{1}{3 \epsilon}, \quad B_{22}\left(p, m_{1}, m_{2}\right) \supset-\frac{1}{4 \epsilon}\left(m_{1}^{2}+m_{2}^{2}+\frac{p^{2}}{3}\right) .
\end{aligned}
$$

In addition, we make use of the algebraic identities

$$
\begin{aligned}
& p^{2} B_{1}\left(p, m_{1}, m_{2}\right)=\frac{1}{2}\left[A\left(m_{1}\right)-A\left(m_{2}\right)-\left(p^{2}-m_{1}^{2}-m_{2}^{2}\right) B_{0}\left(p, m_{1}, m_{2}\right)\right], \\
& p^{2} B_{21}\left(p, m_{1}, m_{2}\right)+d B_{22}\left(p, m_{1}, m_{2}\right)=A\left(m_{2}\right)+m_{1}^{2} B_{0}\left(p, m_{1}, m_{2}\right), \\
& p^{2} B_{21}\left(p, m_{1}, m_{2}\right)+B_{22}\left(p, m_{1}, m_{2}\right)=\frac{1}{2}\left[A\left(m_{2}\right)+\left(m_{1}^{2}-m_{2}^{2}-p^{2}\right) B_{1}\left(p, m_{1}, m_{2}\right)\right],
\end{aligned}
$$


where $A(m)$ is the tadpole form factor

$$
A(m)=\int \frac{\mathrm{d}^{d} k}{i \pi^{2}} \frac{1}{k^{2}-m^{2}+i \varepsilon} .
$$

Lastly, for $m_{1}=m_{2}$ we have the identity

$$
B_{1}(p, m, m)=-\frac{1}{2} B_{0}(p, m, m) .
$$

The three-point form factors are

$$
C_{0 ; \mu ; \mu \nu}=\int \frac{\mathrm{d}^{d} k}{i \pi^{2}} \frac{1 ; k_{\mu} ; k_{\mu} k_{\nu}}{\left(k^{2}-m_{1}^{2}+i \varepsilon\right)\left(\left(k+p_{1}\right)^{2}-m_{2}^{2}+i \varepsilon\right)\left(\left(k+p_{1}+p_{2}\right)^{2}-m_{3}^{2}+i \varepsilon\right)},
$$

where the arguments of $C_{0 ; \mu ; \mu \nu} \equiv C_{0 ; \mu ; \mu \nu}\left(p_{1}, p_{2}, m_{1}, m_{2}, m_{3}\right)$ have been suppressed for notational brevity. We also define scalar form factors via

$$
\begin{aligned}
C_{\mu} & =p_{1 \mu} C_{11}+p_{2 \mu} C_{12}, \\
C_{\mu \nu} & =p_{1 \mu} p_{1 \nu} C_{21}+p_{2 \mu} p_{2 \nu} C_{22}+p_{1(\mu} p_{2 \nu)} C_{23}+\eta_{\mu \nu} C_{24} .
\end{aligned}
$$

The only divergent form factor is $C_{24}$, having the logarithmic divergence

$$
C_{24} \supset \frac{1}{4 \epsilon} \text {. }
$$

Feynman rules. In the chiral basis, the Feynman rules of the model are [43-46]

- To each photon line associate the factor (in the Feynman gauge)

$$
i D_{\mu \nu}(p)=\frac{i \eta_{\mu \nu}}{p^{2}+i \varepsilon}
$$

- To each chiral fermion line associate the factor

$$
i S_{I J}(p)=i \frac{\delta_{I J} \bar{\sigma}_{J} \cdot p+M_{I J}}{p^{2}-M^{2}+i \varepsilon} .
$$

To avoid proliferation of sub- and superscripts, the spinor index assignment, denoted by the lower-case Gothic characters $\mathfrak{a}$ and $\mathfrak{b}$, is understood as follows:

$$
\begin{aligned}
& i S_{L L}(p) \equiv\left[i S_{L L}(p)\right]_{\mathfrak{a} \dot{\mathfrak{b}}}=\frac{i p \cdot \sigma_{\mathfrak{a} \mathfrak{b}}}{p^{2}-M^{2}+i \varepsilon}, \\
& i S_{R R}(p) \equiv\left[i S_{R R}(p)\right]^{\dot{\mathfrak{a}} \mathfrak{b}}=\frac{i p \cdot \bar{\sigma}^{\dot{\mathfrak{a}} \mathfrak{b}}}{p^{2}-M^{2}+i \varepsilon}, \\
& i S_{R L}(p) \equiv\left[i S_{R L}(p)\right]_{\dot{\mathfrak{b}}}^{\dot{\mathfrak{a}}}=\frac{i m_{-} \delta_{\dot{\mathfrak{a}}}^{\dot{\mathfrak{a}}}}{p^{2}-M^{2}+i \varepsilon}, \\
& i S_{L R}(p) \equiv\left[i S_{L R}(p)\right]_{\mathfrak{a}}^{\mathfrak{b}}=\frac{i m_{+} \delta_{\mathfrak{a}}^{\mathfrak{b}}}{p^{2}-M^{2}+i \varepsilon},
\end{aligned}
$$

with

$$
\bar{\sigma}_{L}^{\mu}=\sigma^{\mu} \equiv \sigma_{\mathfrak{a} \mathfrak{b}}^{\mu}, \quad \bar{\sigma}_{R}^{\mu}=\bar{\sigma}^{\mu} \equiv \bar{\sigma}^{\mu, \mathfrak{a} \mathfrak{b}} .
$$

- To each vertex associate a factor of $-i g_{I J} \sigma_{J}^{\mu}$, where $\mathbf{g}=\operatorname{diag}\left(g_{-}, g_{+}\right)$.

- For any closed fermion loop include a factor of -1 and trace over the Lorentz indices.

In the calculation of the one-loop corrections outlined below, we also make heavy use of the product and trace identities of the Pauli matrices, as listed in appendix B of [46]. 
Fermion self-energy. The one-loop chiral fermion self-energies are given by

$$
i \Sigma_{I J}(p)=(-i)^{2} g_{I K} g_{N J} \int \frac{\mathrm{d}^{d} k}{(2 \pi)^{4}} \sigma_{K}^{\mu} i S_{K N}(k+p) \sigma_{N}^{\nu} i D_{\mu \nu}(k),
$$

where we note that the couplings $g_{ \pm}$are dimensionful for $d=4-2 \epsilon$. The numerator is

$$
(2-d) \delta_{K N} \sigma_{N} \cdot k+d M_{K N}
$$

Rewriting in terms of the Passarino-Veltman form factors, we get

$$
\Sigma_{I J}(p)=\frac{1}{16 \pi^{2}} g_{I K} g_{N J}\left[(2-d) \delta_{K N} \sigma_{K} \cdot p B_{1}(p, M, 0)+d M_{K N} B_{0}(p, M, 0)\right] .
$$

Hence, we obtain

$$
\begin{aligned}
\Sigma_{L L} & =\frac{g_{-}^{2}}{16 \pi^{2}}(2-d) \bar{\sigma} \cdot p B_{1}(p, M, 0), \\
\Sigma_{R R} & =\frac{g_{+}^{2}}{16 \pi^{2}}(2-d) \sigma \cdot p B_{1}(p, M, 0), \\
\Sigma_{R L} & =\frac{g_{+} g_{-}}{16 \pi^{2}} d m_{-} B_{0}(p, M, 0), \\
\Sigma_{L R} & =\frac{g_{+} g_{-}}{16 \pi^{2}} d m_{+} B_{0}(p, M, 0) .
\end{aligned}
$$

The full fermion self-energy of the original Dirac field is obtained from the sum over the chiral indices $I$ and $J$ with correct weighting by projection operators. Specifically,

$$
\Sigma=P_{R} \gamma^{0} \Sigma_{L L} P_{L}+P_{L} \gamma^{0} \Sigma_{R R} P_{R}+P_{L} \Sigma_{R L} P_{L}+P_{R} \Sigma_{L R} P_{R}
$$

giving

$$
\Sigma=\left(\begin{array}{ll}
0 & 0 \\
1 & 0
\end{array}\right) \Sigma_{L L}+\left(\begin{array}{ll}
0 & 1 \\
0 & 0
\end{array}\right) \Sigma_{R R}+\left(\begin{array}{ll}
1 & 0 \\
0 & 0
\end{array}\right) \Sigma_{R L}+\left(\begin{array}{ll}
0 & 0 \\
0 & 1
\end{array}\right) \Sigma_{L R} .
$$

Summing these contributions, we obtain the result in eq. (3.3).

Polarization tensor. The polarization tensor is given by

$$
i \Pi^{\mu \nu}(p)=(-1)(-i)^{2} g_{I K} g_{N J} \operatorname{Tr} \int \frac{\mathrm{d}^{d} k}{(2 \pi)^{4}} \sigma_{K}^{\mu} i S_{I J}(p+k) \sigma_{N}^{\nu} i S_{N K}(k) .
$$

Performing the trace over the Lorentz indices, we obtain the numerator

$$
2\left[\left(2 k^{\mu} k^{\nu}+p^{(\mu} k^{\nu)}-\eta^{\mu \nu} k^{2}-\eta^{\mu \nu} p \cdot k\right) \delta_{I J} \delta_{N K}+\eta^{\mu \nu} M_{I J} M_{N K}+i \eta_{I J N K} \varepsilon^{\mu \kappa \nu \lambda}(p+k)_{\kappa} k_{\lambda}\right],
$$

where $\varepsilon^{\mu \kappa \nu \lambda}$ is the Levi-Civita tensor. Here, we have defined $\eta_{I J N K}=1$, if $I=J=K=$ $N=L, \eta_{I J N K}=-1$, if $I=J=N=K=R$, and $\eta_{I J N K}=0$ otherwise.

Rewriting in terms of the Passarino-Veltman form factors, we are left with

$$
\begin{aligned}
\Pi^{\mu \nu}(p)=-\frac{g_{I K} g_{N J}}{4 \pi^{2}}\{ & \left(p^{\mu} p^{\nu}-\eta^{\mu \nu} p^{2}\right)\left(B_{21}+B_{1}\right) \delta_{I J} \delta_{N K} \\
& -\eta^{\mu \nu}\left(M^{2} \delta_{I J} \delta_{N K}-M_{I J} M_{N K}\right) B_{0} \\
& \left.+i \varepsilon^{\mu \kappa \nu \lambda}\left[p_{\kappa} p_{\lambda}\left(B_{21}+B_{1}\right)+\eta_{\kappa \lambda} p^{2} B_{22}\right] \eta_{I J N K}\right\} .
\end{aligned}
$$

When we sum over the chiral indices, the terms proportional to the Levi-Civita tensor cancel, and we obtain the result in eq. (3.8). 
Three-point vertex. The three-point vertices are given by

$$
i \Lambda_{I J}^{\mu}(p, q)=(-i)^{3} g_{I K} g_{N P} g_{Q J} \int \frac{\mathrm{d}^{d} k}{(2 \pi)^{4}} \sigma_{K}^{\nu} i S_{K N}(k) i D_{\nu \lambda}(k+p) \sigma_{P}^{\mu} i S_{P Q}(k+p+q) \sigma_{Q}^{\lambda},
$$

where $p$ and $q$ are the fermion momenta. The numerator is proportional to

$$
\begin{aligned}
&(2-d) {\left[\sigma_{Q}^{\rho} \bar{\sigma}_{P}^{\mu} \sigma_{K}^{\kappa} k_{\kappa}(k+p+q)_{\rho} \delta_{K N} \delta_{P Q}+\sigma_{P}^{\mu} M_{K N} M_{P Q}\right] } \\
&+4\left[k^{\mu} \delta_{K N} M_{P Q}+(k+p+q)^{\mu} M_{K N} \delta_{P Q}\right]
\end{aligned}
$$

such that the vertices can be written

$$
\begin{aligned}
\Lambda_{I J}^{\mu}= & \frac{g_{I K} g_{N P} g_{Q J}}{16 \pi^{2}} \\
& \times\left\{(2-d)\left[\left((2-d) \sigma_{P}^{\mu} C_{24}+\sigma_{Q}^{\rho} \bar{\sigma}_{P}^{\mu} \sigma_{K}^{\kappa} F_{\kappa \rho}\right) \delta_{K N} \delta_{P Q}+\sigma_{P}^{\mu} M_{K N} M_{P Q}\right]\right. \\
& \left.+4\left(p^{\mu} C_{11}+q^{\mu} C_{12}\right)\left(\delta_{K N} M_{P Q}+M_{K N} \delta_{P Q}\right)+4(p+q)^{\mu} C_{0} M_{K N} \delta_{P Q}\right\} .
\end{aligned}
$$

Herein, we have defined

$$
F_{\kappa \rho}=p_{\kappa} p_{\rho}\left(C_{11}+C_{21}\right)+q_{\kappa} q_{\rho}\left(C_{22}+C_{12}\right)+p_{\kappa} q_{\rho}\left(C_{23}+C_{11}\right)+q_{\kappa} p_{\rho}\left(C_{23}+C_{12}\right) .
$$

The three-point form factors are evaluated at $p_{1}=p, p_{2}=q, m_{1}=m_{3}=M$ and $m_{2}=0$. Hence, element by element, we find that

$$
\begin{aligned}
\Lambda_{L L}^{\mu} & =\frac{g_{-}^{2}}{16 \pi^{2}}(2-d)\left[g_{-}\left((2-d) \bar{\sigma}^{\mu} C_{24}+\bar{\sigma}^{\rho} \sigma^{\mu} \bar{\sigma}^{\kappa} F_{\kappa \rho}\right)+g_{+} \bar{\sigma}^{\mu} M^{2} C_{0}\right] \\
\Lambda_{R R}^{\mu} & =\frac{g_{+}^{2}}{16 \pi^{2}}(2-d)\left[g_{+}\left((2-d) \sigma^{\mu} C_{24}+\sigma^{\rho} \bar{\sigma}^{\mu} \sigma^{\kappa} F_{\kappa \rho}\right)+g_{-} \sigma^{\mu} M^{2} C_{0}\right], \\
\Lambda_{R L}^{\mu} & =\frac{g_{+} g_{-}}{4 \pi^{2}} m_{-}\left[\left(g_{+}+g_{-}\right)\left(p^{\mu} C_{11}+q^{\mu} C_{12}\right)+g_{-}\left(p^{\mu}+q^{\mu}\right) C_{0}\right], \\
\Lambda_{L R}^{\mu} & =\frac{g_{+} g_{-}}{4 \pi^{2}} m_{+}\left[\left(g_{+}+g_{-}\right)\left(p^{\mu} C_{11}+q^{\mu} C_{12}\right)+g_{+}\left(p^{\mu}+q^{\mu}\right) C_{0}\right] .
\end{aligned}
$$

As in the case of the self-energy, we have

$$
\Lambda^{\mu}=\left(\begin{array}{ll}
0 & 0 \\
1 & 0
\end{array}\right) \Lambda_{L L}^{\mu}+\left(\begin{array}{ll}
0 & 1 \\
0 & 0
\end{array}\right) \Lambda_{R R}^{\mu}+\left(\begin{array}{ll}
1 & 0 \\
0 & 0
\end{array}\right) \Lambda_{R L}^{\mu}+\left(\begin{array}{ll}
0 & 0 \\
0 & 1
\end{array}\right) \Lambda_{L R}^{\mu} .
$$

Summing over the contributions, we obtain the result for the total vertex in eq. (3.11).

Open Access. This article is distributed under the terms of the Creative Commons Attribution License (CC-BY 4.0), which permits any use, distribution and reproduction in any medium, provided the original author(s) and source are credited.

\section{References}

[1] G. Barenboim, L. Borissov, J.D. Lykken and A.Y. Smirnov, Neutrinos as the messengers of CPT violation, JHEP 10 (2002) 001 [hep-ph/0108199] [INSPIRE].

[2] C.M. Bender, Making sense of non-Hermitian Hamiltonians, Rept. Prog. Phys. 70 (2007) 947 [hep-th/0703096] [INSPIRE].

[3] P. Dorey, C. Dunning and R. Tateo, The ODE/IM Correspondence, J. Phys. A 40 (2007) R205 [hep-th/0703066] [INSPIRE]. 
[4] C.M. Bender, D.C. Brody and H.F. Jones, Scalar quantum field theory with cubic interaction, Phys. Rev. Lett. 93 (2004) 251601 [hep-th/0402011] [INSPIRE].

[5] C.M. Bender and K.A. Milton, A nonunitary version of massless quantum electrodynamics possessing a critical point, J. Phys. A 32 (1999) L87 [InSPIRE].

[6] K. Johnson, M. Baker and R.S. Willey, Quantum Electrodynamics, Phys. Rev. Lett. 11 (1963) 518 [INSPIRE].

[7] K. Johnson, M. Baker and R. Willey, Selfenergy of the electron, Phys. Rev. 136 (1964) B1111 [INSPIRE].

[8] K. Johnson, R. Willey and M. Baker, Vacuum polarization in quantum electrodynamics, Phys. Rev. 163 (1967) 1699 [InSPIRE].

[9] C.M. Bender, I. Cavero-Pelaez, K.A. Milton and K.V. Shajesh, PT-symmetric quantum electrodynamics, Phys. Lett. B 613 (2005) 97 [hep-th/0501180] [INSPIRE].

[10] K. Jones-Smith and H. Mathur, A New Class of non-Hermitian Quantum Hamiltonians with PT Symmetry, Phys. Rev. A 82 (2010) 042101 [arXiv:0908.4255] [INSPIRE].

[11] C.M. Bender and S.P. Klevansky, PT-Symmetric Representations of Fermionic Algebras, Phys. Rev. A 84 (2011) 024102 [arXiv:1104.4156] [InSPIRE].

[12] K. Jones-Smith and H. Mathur, Relativistic Non-Hermitian Quantum Mechanics, Phys. Rev. D 89 (2014) 125014 [arXiv:0908.4257] [INSPIRE].

[13] Particle Data Group collaboration, K.A. Olive et al., Review of Particle Physics, Chin. Phys. C 38 (2014) 090001 [inSPIRE].

[14] B. Pontecorvo, Inverse beta processes and nonconservation of lepton charge, Sov. Phys. JETP 7 (1958) 172 [inSPIRE].

[15] Z. Maki, M. Nakagawa and S. Sakata, Remarks on the unified model of elementary particles, Prog. Theor. Phys. 28 (1962) 870 [InSPIRE].

[16] N. Cabibbo, Unitary Symmetry and Leptonic Decays, Phys. Rev. Lett. 10 (1963) 531 [INSPIRE].

[17] M. Kobayashi and T. Maskawa, CP Violation in the Renormalizable Theory of Weak Interaction, Prog. Theor. Phys. 49 (1973) 652 [InSPIRE].

[18] A.D. Sakharov, Violation of CP Invariance, C Asymmetry and Baryon Asymmetry of the Universe, Pisma Zh. Eksp. Teor. Fiz. 5 (1967) 32 [JETP Lett. 5 (1967) 24] [Sov. Phys. Usp. 34 (1991) 392] [Usp. Fiz. Nauk 161 (1991) 61] [INSPIRE].

[19] M. Fukugita and T. Yanagida, Baryogenesis Without Grand Unification, Phys. Lett. B 174 (1986) 45 [INSPIRE].

[20] A. Pilaftsis, Heavy Majorana neutrinos and baryogenesis, Int. J. Mod. Phys. A 14 (1999) 1811 [hep-ph/9812256] [INSPIRE].

[21] W. Buchmüller, R.D. Peccei and T. Yanagida, Leptogenesis as the origin of matter, Ann. Rev. Nucl. Part. Sci. 55 (2005) 311 [hep-ph/0502169] [INSPIRE].

[22] S. Davidson, E. Nardi and Y. Nir, Leptogenesis, Phys. Rept. 466 (2008) 105 [arXiv: 0802.2962] [INSPIRE].

[23] S. Blanchet and P. Di Bari, The minimal scenario of leptogenesis, New J. Phys. 14 (2012) 125012 [arXiv: 1211.0512] [INSPIRE]. 
[24] P. Minkowski, $\mu \rightarrow e \gamma$ at a Rate of One Out of $10^{9}$ Muon Decays?, Phys. Lett. B 67 (1977) 421 [INSPIRE].

[25] R.N. Mohapatra and G. Senjanović, Neutrino Mass and Spontaneous Parity Violation, Phys. Rev. Lett. 44 (1980) 912 [INSPIRE].

[26] M. Gell-Mann, P. Ramond and R. Slansky, Complex Spinors and Unified Theories, Conf. Proc. C 790927 (1979) 315 [arXiv:1306.4669] [INSPIRE].

[27] T. Yanagida, Horizontal symmetry and masses of neutrinos, Conf. Proc. C 7902131 (1979) 95 [INSPIRE].

[28] J. Schechter and J.W.F. Valle, Neutrino Masses in $\mathrm{SU}(2) \times \mathrm{U}(1)$ Theories, Phys. Rev. D 22 (1980) 2227 [INSPIRE].

[29] V.A. Kuzmin, V.A. Rubakov and M.E. Shaposhnikov, On the Anomalous Electroweak Baryon Number Nonconservation in the Early Universe, Phys. Lett. B 155 (1985) 36 [INSPIRE].

[30] C.M. Bender, H.F. Jones and R.J. Rivers, Dual PT-symmetric quantum field theories, Phys. Lett. B 625 (2005) 333 [hep-th/0508105] [INSPIRE].

[31] J. Alexandre and C.M. Bender, Foldy-Wouthuysen transformation for non-Hermitian Hamiltonians, J. Phys. A 48 (2015) 185403 [arXiv:1501.01232] [INSPIRE].

[32] C.M. Bender and T.T. Wu, Anharmonic oscillator, Phys. Rev. 184 (1969) 1231 [InSPIRE].

[33] G. Passarino and M.J.G. Veltman, One Loop Corrections for $e^{+} e^{-}$Annihilation Into $\mu^{+} \mu^{-}$ in the Weinberg Model, Nucl. Phys. B 160 (1979) 151 [INSPIRE].

[34] R.N. Mohapatra and J.C. Pati, Left-Right Gauge Symmetry and an Isoconjugate Model of CP-violation, Phys. Rev. D 11 (1975) 566 [INSPIRE].

[35] R.N. Mohapatra and J.C. Pati, A Natural Left-Right Symmetry, Phys. Rev. D 11 (1975) 2558 [INSPIRE].

[36] G. Senjanović and R.N. Mohapatra, Exact Left-Right Symmetry and Spontaneous Violation of Parity, Phys. Rev. D 12 (1975) 1502 [inSPIRE].

[37] P.S. Bhupal Dev, P. Millington, A. Pilaftsis and D. Teresi, Flavour Covariant Transport Equations: an Application to Resonant Leptogenesis, Nucl. Phys. B 886 (2014) 569 [arXiv: 1404.1003] [INSPIRE].

[38] M. Drewes, The Phenomenology of Right Handed Neutrinos, Int. J. Mod. Phys. E 22 (2013) 1330019 [arXiv: 1303.6912] [INSPIRE].

[39] F.F. Deppisch, P.S. Bhupal Dev and A. Pilaftsis, Neutrinos and Collider Physics, New J. Phys. 17 (2015) 075019 [arXiv: 1502.06541] [INSPIRE].

[40] J. Alexandre, J. Brister and N. Houston, On higher-order corrections in a four-fermion Lifshitz model, Phys. Rev. D 86 (2012) 025030 [arXiv:1204.2246] [INSPIRE].

[41] J. Alexandre, J. Leite and N.E. Mavromatos, Lorentz-violating regulator gauge fields as the origin of dynamical flavor oscillations, Phys. Rev. D 87 (2013) 125029 [arXiv:1304.7706] [INSPIRE].

[42] J. Alexandre, J. Leite and N.E. Mavromatos, Quasirelativistic fermions and dynamical flavor oscillations, Phys. Rev. D 90 (2014) 045026 [arXiv:1404.7429] [INSPIRE]. 
[43] C. Bouchiat and L. Michel, Mesure de la polarisation des electrons relativistes, Nucl. Phys. 5 (1958) 416.

[44] L. Michel, Covariant description of polarization, Nuovo Cim. Suppl. 14 (1959) 95.

[45] H.E. Haber, Spin Formalism and Applications to New Physics Searches, in proceedings of the 21st SLAC Summer Institute on Particle Physics: "Spin Structure in High Energy Processes", (1993) pg. 231.

[46] H.K. Dreiner, H.E. Haber and S.P. Martin, Two-component spinor techniques and Feynman rules for quantum field theory and supersymmetry, Phys. Rept. 494 (2010) 1 [arXiv:0812.1594] [INSPIRE]. 